\title{
Implementation of spectrogram for an improved EMG-based functional capacity evaluation's core-lifting task
}

\begin{abstract}
This paper proposes a technique to automatically categorize work levels categories to improve the conventional functional capacity evaluation's core lifting task. Surface EMG signals were collected from biceps brachii and erector spinae muscles. Spectrogram was used as a pre-processing approach for auto-segmentation of the EMG signal and for the feature extraction. This set of features was extracted to accurately differentiate between a medium work level and heavy work level. These features were then reduced using linear discriminant analysis and support vector machine acts as a classifier. The results showed that the proposed system offered excellent performance in classifying the work levels categories with high accuracy, sensitivity, specificity, and zero cross-validation error.
\end{abstract}

Keyword: Electromyography (EMG); Functional capacity evaluation; Lifting task; Timefrequency distribution; Spectrogram; Linear discriminant analysis (LDA); Support vector machine (SVM) 